IRRITABLE BOWEL SYNDROME

\title{
Downregulation of epithelial apoptosis and barrier repair in active Crohn's disease by tumour necrosis factor $\alpha$ antibody treatment
}

\author{
S Zeissig, C Bojarski, N Buergel, J Mankertz, M Zeitz, M Fromm, J D Schulzke
}

Gut 2004;53:1295-1302. doi: 10.1136/gut.2003.036632

See end of article for authors' affiliations

\section{Correspondence to:} Professor J D Schulzke, Medizinische Klinik I, Charitè-Universitary Medicine Berlin, Campus Benjamin Franklin, Hindenburgdamm 30 Berlin 12200, Germany; joerg.schulzke@charite.de

Revised version received 3 February 2004 Accepted for publication 17 February 2004

\begin{abstract}
Background and aims: Barrier dysfunction is an important feature contributing to inflammation and diarrhoea in Crohn's disease (CD). Recently, tumour necrosis factor $\alpha$ (TNF- $\alpha$ ) antibodies were recognised as effective in steroid refractory $C D$. The aim of this study was to characterise the effects of this therapy on the epithelial barrier.

Patients and methods: Forceps biopsies were obtained from the sigmoid colon before and 14 days after TNF- $\alpha$ antibody therapy in 11 patients treated for chronic active CD (Crohn's disease activity index >150). Epithelial apoptoses were measured after terminal deoxynucleotidyl transferase mediated deoxyuridine triphosphate nick end labelling (TUNEL) and 4',6-diamidino-2-phenylindole staining. Epithelial resistance was determined by alternating current impedance analysis in miniaturised Ussing chambers. Occludin, claudin 1, and claudin 4 expression was quantified in immunoblots.

Results: The epithelial apoptotic ratio was $2.1(0.2) \%$ in controls and increased to $5.3(1.0) \%$ in CD. TNF- $\alpha$ antibody therapy decreased the apoptotic ratio to 2.9 (1.0)\% (normalised in 10 of 11 patients). In parallel, epithelial resistance was lower in CD than in controls (24 (3) v 42 (3) $\Omega \times \mathrm{cm}^{2}$ ) and improved to 34 (3) $\Omega \times \mathrm{cm}^{2}$ after therapy. Occludin, claudin 1, and claudin 4 were not affected by TNF- $\alpha$ antibody therapy. In support of a functional role of epithelial apoptoses in CD, a similar decrease in resistance of $-40 \%$ was observed when the apoptotic rate was selectively upregulated from $2.6 \%$ to $5.4 \%$ with camptothecin in HT-29/B6 cells.

Conclusions: Epithelial apoptoses were upregulated in the colon in CD and restored to normal in 10 of 11 patients by TNF- $\alpha$ antibody therapy. This is the structural correlate of epithelial barrier dysfunction measured as epithelial resistance while expression of tight junction proteins did not contribute to this therapeutic effect.
\end{abstract}

rohn's disease $(C D)$ is a chronic inflammatory bowel disease with segmental inflammation throughout the gastrointestinal tract. Even though corticosteroids are often efficient in controlling symptoms of mild CD, ${ }^{1}$ a minor proportion of $\mathrm{CD}$ patients are resistant to both standard therapy and combinations with purine antimetabolites or methotrexate. In these patients, tumour necrosis factor $\alpha$ $(\mathrm{TNF}-\alpha)$ antibody therapy with the chimeric monoclonal TNF- $\alpha$ antibody infliximab has been shown to be highly effective. $^{23}$

Epithelial barrier function comprising fencing properties against small ions as well as larger molecules as antigens has been shown to be seriously impaired in $\mathrm{CD}^{4}{ }^{5}$ The proinflammatory cytokine TNF- $\alpha$, which is elevated in patients with $\mathrm{CD}^{6}{ }^{6}$ is thought to play a central role in this barrier defect. In studies with model epithelia such as HT-29/B6, TNF- $\alpha$ reduced epithelial barrier function by affecting both induction of single cell apoptosis ${ }^{7}$ and the epithelial tight junction. The latter effect was indicated by a TNF- $\alpha$ dependent decrease in tight junction strands on freeze fracture electron microscopy ${ }^{8}$ and a decrease in expression of tight junction proteins. ${ }^{9}$ For the tight junction protein occludin, this was characterised as regulation of expression via the occludin promoter, as obtained from reporter gene assays. ${ }^{9}$ Finally, this was also corroborated in inflammatory bowel disease epithelia where tight junction alterations and apoptotic foci were found to contribute to the barrier defect in ulcerative colitis. ${ }^{10}$

Hence it is not surprising that targeting TNF- $\alpha$ with antibody therapy could improve intestinal barrier function.
Recently, Suenaert et al have demonstrated repair of intestinal barrier function by an in vivo permeability test. ${ }^{11}$ However, to date it is not known which barrier features and mechanisms are involved in this TNF- $\alpha$ antibody effect in CD. Therefore, in the present study, our aim was to characterise the mechanisms of barrier dysfunction and repair in CD.

In recent studies, dysregulation of immune cell apoptosis has been found to be a major factor in impairment of intestinal barrier function in CD. T lymphocytes, an important source of proinflammatory cytokines, were shown to be resistant to apoptotic stimuli in CD. ${ }^{12-14}$ However, after TNF- $\alpha$ antibody therapy, both lamina propria T lymphocytes ${ }^{15}$ and monocytes ${ }^{16}$ underwent upregulation of apoptosis. Therefore, the question arose whether or not enterocyte apoptosis is also upregulated by TNF- $\alpha$ antibody therapy, either as the result of a direct reduction of circulating proapoptotic TNF- $\alpha$ or indirectly as a consequence of immune cell eradication.

In the present study, apoptosis of colonic epithelial cells and tight junction protein expression were examined in CD patients before and after TNF- $\alpha$ therapy in relation to functional changes in the epithelial barrier, as obtained from alternating current impedance analysis on colonic biopsies

Abbreviations: TNF- $\alpha$, tumour necrosis factor $\alpha$; DAPI, $4^{\prime}, 6$ diamidino-2-phenylindole; $R^{e}$, epithelial electrical wall resistance; $R^{\text {sub }}$, subepithelial electrical wall resistance; $R^{\dagger}$, transmural wall resistance; $C D$, Crohn's disease; TUNEL, terminal deoxynucleotidyl transferase mediated deoxyuridine triphosphate nick end labelling; CDAl, Crohn's disease activity index 
studied in vitro. In contrast with immune cell apoptosis, epithelial apoptosis was found to be downregulated while tight junction protein expression was not significantly affected within the two week time period after therapy.

\section{PATIENTS AND METHODS \\ Patients}

Biopsies from the distal colon $(30 \mathrm{~cm} a b a n o)$ of 11 patients with steroid refractory chronic active CD and an inflamed distal colon were investigated before and 14 days after TNF- $\alpha$ antibody therapy (with infliximab $5 \mathrm{mg} / \mathrm{kg}$ body weight intravenously). CD activity index (CDAI) was evaluated according to Best and colleagues. ${ }^{17}$ Control biopsies were obtained from eight patients investigated for tumour exclusion who did not show inflammation macroscopically or microscopically. Prednisolone of more than $10 \mathrm{mg}$ /day (or equivalent) was not allowed during the last 14 days before biopsy. Administration of other medications (for example, sulphasalazine or 5-aminosalicylic acid) was continued. The investigation was approved by the local ethics committee.

\section{Histology and apoptotic ratio}

Biopsies were obtained endoscopically, fixed in $4 \%$ formalin, and embedded in paraffin for morphological analysis. Serial sections $(3 \mu \mathrm{m})$ were used for immunofluorescence detection of epithelial apoptoses. Cellular DNA was stained with $4^{\prime}, 6^{\prime}$-diamidino-2' -phenylindoladihydrochloride (DAPI) ${ }^{18}$ or TUNEL (terminal deoxynucleotidyl transferase (TdT) mediated deoxyuridine triphosphate (dUTP) nick end labelling) (Roche, Mannheim, Germany), respectively. Typical apoptotic changes and criteria for evaluation comprised condensation of chromatin, its compaction along the periphery of the nucleus, and segmentation of the nucleus. The rate of apoptosis was determined as the percentage of apoptotic nuclei per high power field. An average of six fields $(\sim 150$ enterocytes/field $)$ in $11+8$ samples per condition were counted.

\section{Tissue preparation for electrophysiological investigation}

Biopsies were obtained endoscopically with a $3.9 \mathrm{~mm}$ forceps using a colonoscope 1T-20 (Olympus Inc., Tokyo, Japan). Specimens were spread out under a dissection microscope. A perforated plastic disc with an inner diameter of $2.5 \mathrm{~mm}$ was glued to the serosal side of the biopsy using histoacryl tissue glue (B Braun, Melsungen, Germany). Subsequently, this disc was placed in a micro container tightened with silicon rubber seals and mounted in Ussing-type chambers, as described previously. ${ }^{19}$ Tissues were transported to the laboratory on ice in oxygenated bathing solution. The time between taking the biopsies and mounting the tissues into Ussing chambers was approximately 20-30 minutes.

\section{Solutions and drugs}

The bathing solution for the Ussing experiments contained (in $\mathrm{mM}$ ): $\mathrm{Na}^{+} 140, \mathrm{Cl}^{-} 123.8, \mathrm{~K}^{+} 5.4, \mathrm{Ca}^{2+} 1.2, \mathrm{Mg}^{2+} 1.2$, $\mathrm{HPO}_{4}{ }^{2-} 2.4, \mathrm{H}_{2} \mathrm{PO}_{4}{ }^{-} 0.6, \mathrm{HCO}_{3}{ }^{-} 2 \mathrm{l}, \mathrm{D}(+)$-glucose $10, \beta-\mathrm{OH}-$ butyrate 0.5 , glutamine 2.5 , and $\mathrm{D}(+)$-mannose $10 .^{20}$ Amiloride $(10 \mu \mathrm{M})$ was present on both sides of the Ussing chamber throughout the experiments. The solution was gassed with $95 \% \quad \mathrm{O}_{2}$ and $5 \% \quad \mathrm{CO}_{2}$. Temperature was maintained at $37^{\circ} \mathrm{C}$ using water jacketed reservoirs. The $\mathrm{pH}$ was 7.4 in all experiments. Antibiotics (azlocillin $50 \mathrm{mg} / \mathrm{l}$ and tobramycin $4 \mathrm{mg} / \mathrm{l}$ ) served to prevent bacterial growth and had no effect on $\mathrm{I}_{\mathrm{SC}}$ at the concentrations used. ${ }^{21}$

\section{Alternating current impedance analysis}

Ussing-type experiments were performed as described previously in miniaturised Ussing chambers using a computer controlled voltage clamp device (CVC 6; Fiebig, Berlin, Germany). ${ }^{22}$ Alternating current impedance analysis can differentiate epithelial $\left(\mathrm{R}^{\mathrm{e}}\right)$ and subepithelial $\left(\mathrm{R}^{\text {sub }}\right)$ portions of transmural wall resistance $\left(\mathrm{R}^{\mathrm{t}}\right)$ based on the three parameter model of the colonic wall..$^{23}$ In this model, the epithelium is described as an electrical equivalent circuit by a resistor and a capacitor in parallel and the subepithelium by a resistor in series. After application of 48 discrete frequencies of an effective sine wave alternating current of $35 \mu \mathrm{A} / \mathrm{cm}^{2}$ ranging from $1.3 \mathrm{~Hz}$ to $65 \mathrm{kHz}$, changes in tissue voltage were detected by phase sensitive amplifiers (1250 frequency response analyser and 1286 electrochemical interface; Solartron Schlumberger, Farnborough, Hampshire, UK). Complex impedance values were calculated and corrected for the resistance of the bathing solution and the frequency behaviour of the measuring device. Then, for each tissue, the impedance locus was plotted on a Nyquist diagram and a circle segment was fitted by least squares analysis. Due to the frequency dependent electrical characteristics of the capacitor, transmural resistance $\left(\mathrm{R}^{\mathrm{t}}\right)$ was obtained at low frequencies whereas subepithelial resistance $\left(\mathrm{R}^{\mathrm{sub}}\right)$ was obtained at high frequencies. Epithelial resistance $\left(\mathrm{R}^{\mathrm{e}}\right)$ was obtained from $\mathrm{R}^{\mathrm{e}}=\mathrm{R}^{\mathrm{t}}-\mathrm{R}^{\mathrm{sub}}$.

\section{Western blot analysis}

To determine tight junction protein expression, western blot analysis was performed from membrane extracts of colonic biopsy specimens. Tissues of sigmoid colon obtained at colonoscopy before and two weeks after infliximab treatment were homogenised (by douncing) on iced lysate buffer containing $20 \mathrm{mM}$ Tris $\mathrm{pH} 7.4,5 \mathrm{mM} \mathrm{MgCl}_{2}, 1 \mathrm{mM}$ EDTA, $0.3 \mathrm{mM}$ EGTA, $1 \mu \mathrm{l} / \mathrm{ml}$ aprotinin, $16 \mu \mathrm{g} / \mathrm{ml}$ benzamidine- $\mathrm{HCl}$, $10 \mu \mathrm{g} / \mathrm{ml}$ phenanthroline, $10 \mu \mathrm{g} / \mathrm{ml}$ leupeptin, $10 \mu \mathrm{g} / \mathrm{ml}$ pepstatin, $2 \mathrm{mM}$ PMSF, $210 \mu \mathrm{g} / \mathrm{ml}$ sodium fluoride, $2.16 \mathrm{mg} / \mathrm{ml}$ $\beta$-glycerophosphate, $18.4 \mu \mathrm{g} / \mathrm{ml} \mathrm{NaVO}$, and $1 \mu \mathrm{l} / \mathrm{ml}$ trypsin inhibitor (all substances obtained from Sigma Chemicals, St Louis, Missouri, USA). Membrane fractions were obtained by passage through a 26 gauge $1 / 2$ needle. To remove insoluble material the extract was centrifuged at $350 \mathrm{~g}$ for five minutes at $4{ }^{\circ} \mathrm{C}$. The supernatant was then centrifuged at $43000 \mathrm{~g}$ for 30 minutes at $4^{\circ} \mathrm{C}$. The pellet representing a crude membrane fraction was resuspended in lysate buffer. Protein concentrations were determined by Pierce BCA assay. Aliquots of $2.5 \mu \mathrm{g}$ were separated by polyacrylamide gel electrophoresis (8.5\% for occludin and $12.5 \%$ for claudins) and transferred to a polyscreen PVDF transfer membrane (NEN Life Science Products, Boston, Massachusetts, USA). Blots were blocked for two hours in $5 \%$ milk powder and then overnight in 5\% bovine serum albumin (at $4^{\circ} \mathrm{C}$ ) before incubation with primary rabbit polyclonal IgG antibodies directed against claudin 1 and occludin and with primary mouse monoclonal IgG antibodies directed against claudin 4. POD conjugated goat antirabbit IgG or goat antimouse IgG antibodies and the chemiluminescence detection system Lumi-Light Western Blotting Kit (Roche, Mannheim, Germany) were used to detect bound antibodies. Chemiluminescence signals were detected using a LAS-1000 imaging system (Fuji, Tokyo, Japan) and analysed with the AIDA program package (Raytest, Berlin, Germany). Densitometric analysis of protein expression before and two weeks after infliximab was always performed on the same blot for each individual patient.

\section{Induction of apoptosis in HT-29/B6 cells}

HT-29/B6 cells, which are subcloned from the human colon carcinoma cell line HT-29, ${ }^{24}$ grow as highly differentiated polarised monolayers. HT-29/B6 cells were routinely cultured in $25 \mathrm{~cm}^{2}$ culture flasks in RPMI 1640 (Biochrom, Berlin, Germany) containing $2 \%$ stabilised L-glutamine and supplemented with $10 \%$ fetal calf serum at $37^{\circ} \mathrm{C}$ in an atmosphere of 

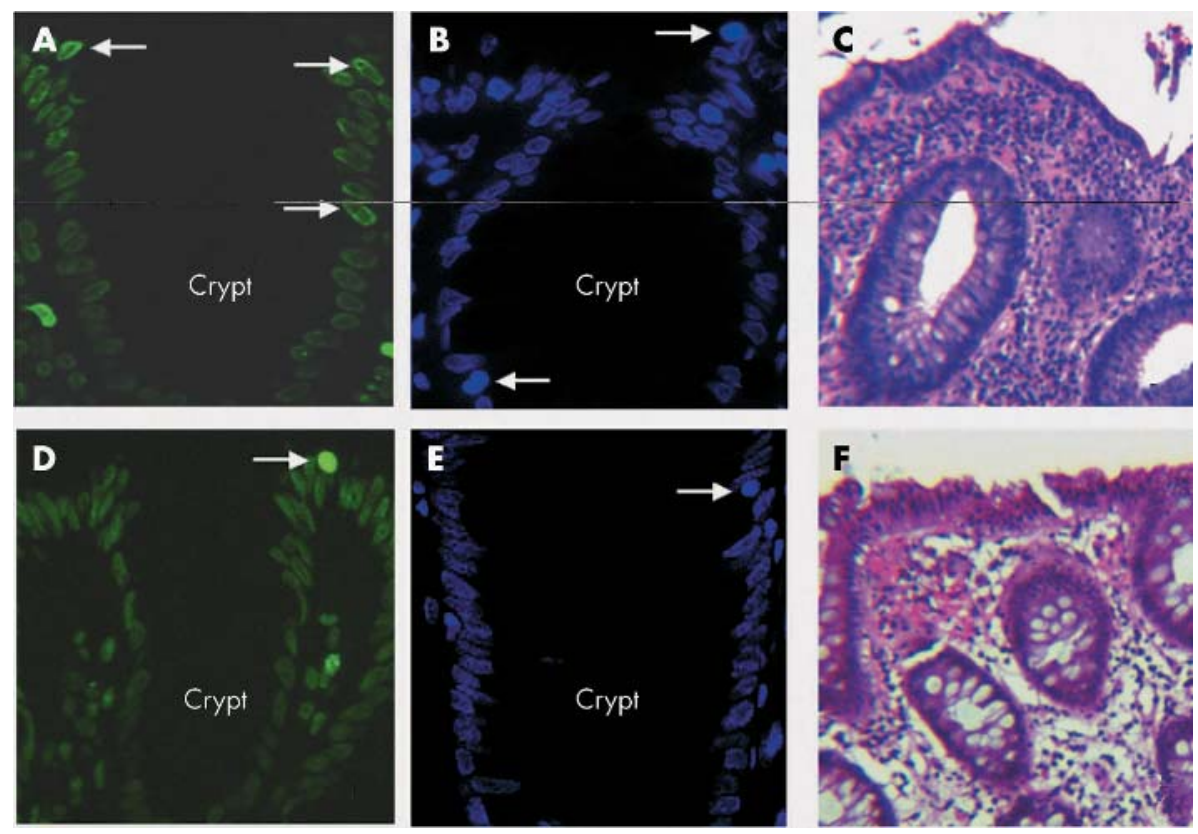

Figure 1 Terminal deoxynucleotidyl transferase mediated deoxyuridine triphosphate nick end labelling (TUNEL) (A, D), 4",6-diamidino-2phenylindole $(B, E)$, and haematoxylin-eosin $(C, F)$ stained thin sections of sigmoid colon from one representative Crohn's disease patient before $(A, B, C)$ and two weeks after (D, E, F) tumour necrosis factor $\alpha$ antibody therapy. Arrows indicate apoptotic enterocytes (magnification $200 \times)$.

$95 \% \mathrm{O}_{2}$ and $5 \% \mathrm{CO}_{2}$. For electrophysiological measurements, cells were seeded on Millicell PCF filters (effective area $0.6 \mathrm{~cm}^{2}$; Millipore) with an average concentration of $7 \times 10^{5}$ cells $/ \mathrm{cm}^{2}$. Three filters were placed together into one conventional culture dish (OD $60 \mathrm{~mm}$ ) filled with $10 \mathrm{ml}$ of culture medium. Confluence of the monolayers was reached after seven days. On day 7, confluent monolayers of HT-29/B6 cells were incubated serosally with the topoisomerase $I$ inhibitor camptothecin at varying concentrations for 48 hours. For quantification of apoptosis, monolayers were fixed with $4 \%$ formalin, embedded in paraffin, and TUNEL stained.

Transepithelial resistance $\left(\mathrm{R}^{\mathrm{t}}\right)$ of the monolayers was measured by a modification of the method described by Kreusel and colleagues. ${ }^{24}$ Briefly, electrical measurements were performed in culture dishes by two fixed pairs of
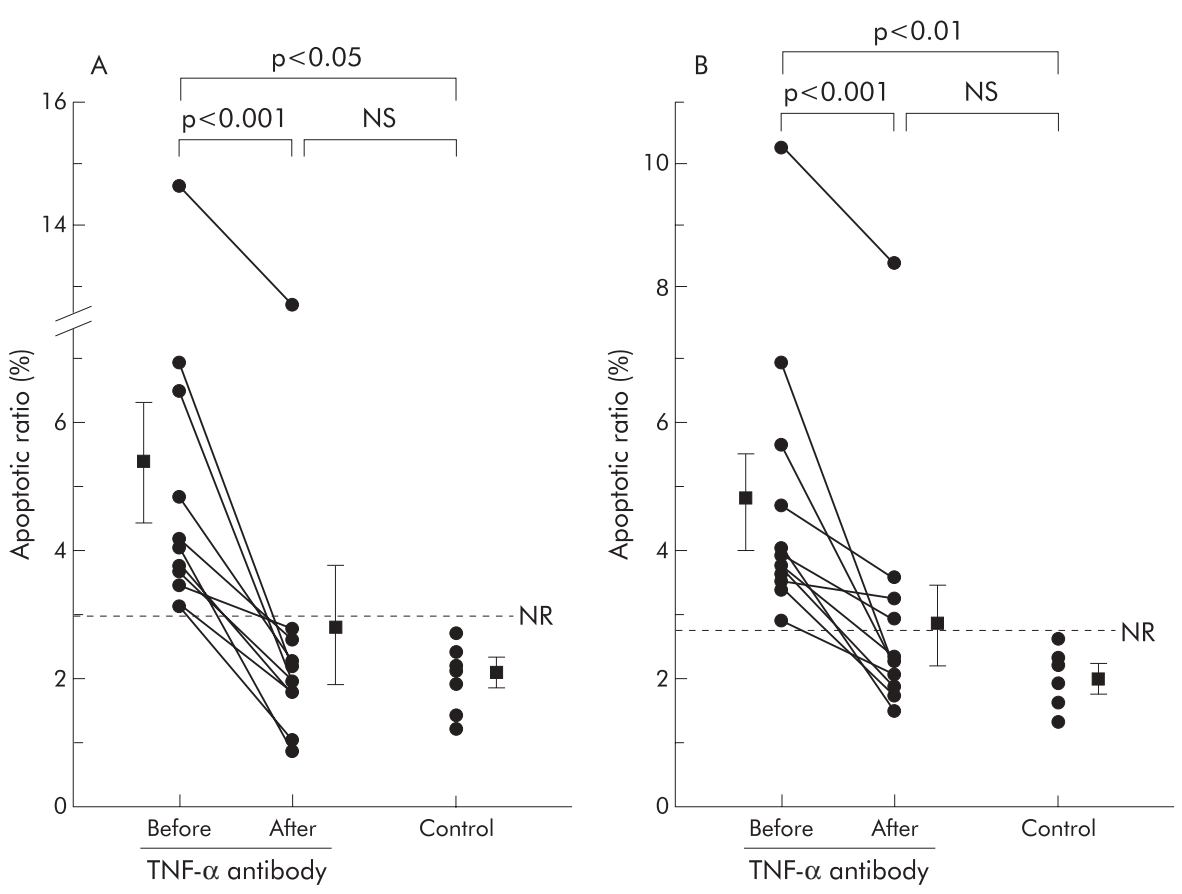

Figure 2 Apoptotic ratio of controls $(n=8)$ and Crohn's disease patients $(n=11)$ before and two weeks after fumour necrosis factor $\alpha$ (TNF- $\alpha$ ) antibody treatment, as determined in terminal deoxynucleotidyl transferase mediated deoxyuridine triphosphate nick end labelling (TUNEL) stained sections (A) and 4',6-diamidino-2-phenylindole stained sections (B), respectively. Individual data are given as means (SEM). Upper limit of the normal range (NR), as obtained from the mean of control $\pm 2 S D$, is indicated by the broken line. 
electrodes (STX-2; World Precision Instruments, USA) connected with an impedance meter (D Sorgenfrei, Inst Klinische Physiologie). $\mathrm{R}^{\mathrm{t}}$ was calculated from the voltage deflections caused by an external $\pm 10 \mu \mathrm{A}, 21 \mathrm{~Hz}$ rectangular current. Depth of immersion and position of the filters was standardised mechanically. Temperature was maintained at $37^{\circ} \mathrm{C}$ during the measurements by a temperature controlled warming plate. Resistance values were corrected for the resistance of the empty filter and of the bathing solution. The set up was placed under a plastic hood, and the electrodes were regularly disinfected with $70 \%$ ethanol. This proved to be sufficient to allow repetitive measurements in individual dishes over several days without infection.

\section{Statistical analysis}

The SPSS for Windows software package was applied. All data are means (SEM). Differences between groups were tested by analysis of variance (one way ANOVA; least significant difference). For comparison of results of $C D$
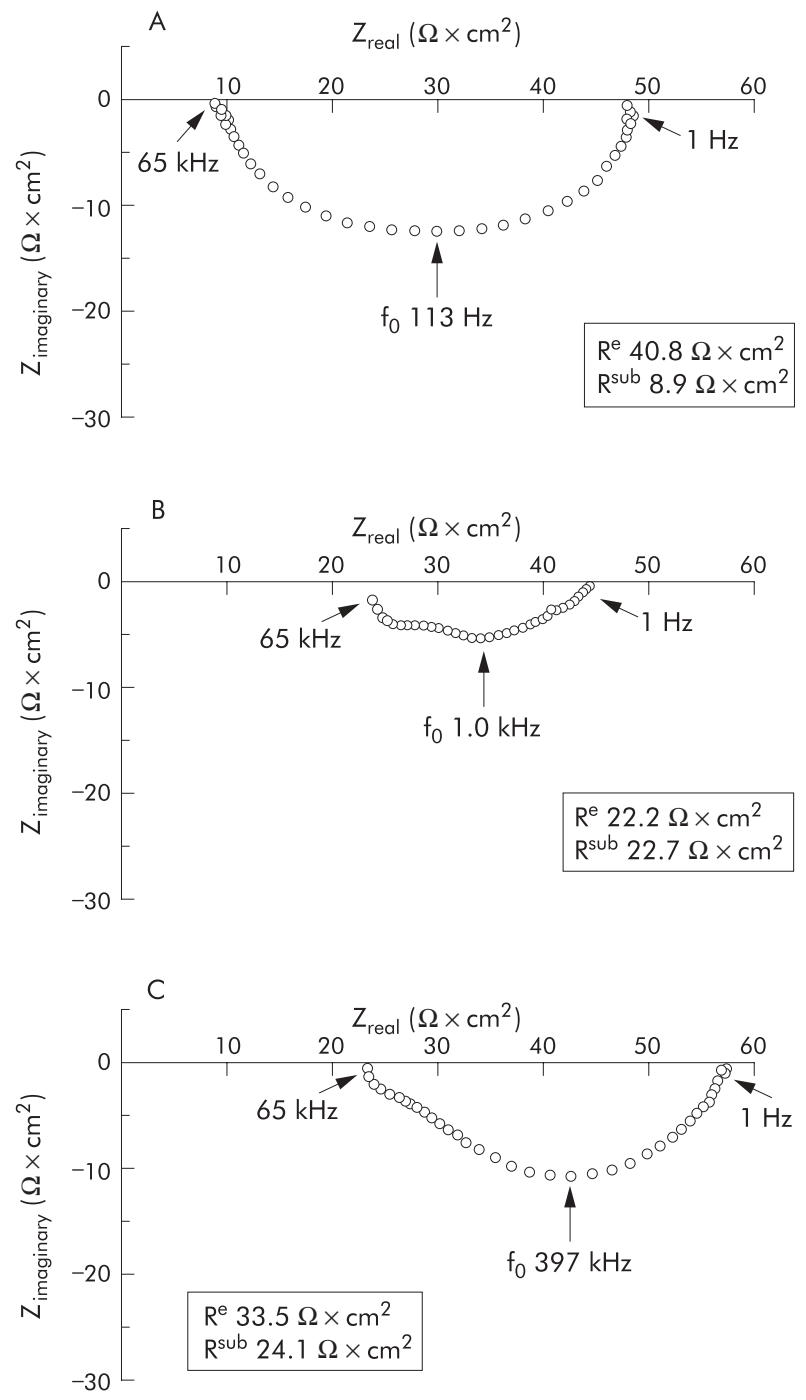

Figure 3 Original impedance locus plots of human sigmoid colon. (A) Control. (B) Crohn's disease (CD) before tumour necrosis factor $\alpha$ (TNF- $\alpha$ ) antibody treatment. (C) CD after TNF- $\alpha$ antibody treatment. $Z_{\text {real }}$ gives the ohmic component and $Z_{\text {imaginary }}$ the reactive component of the complex impedance. Intersections between the semi circle and $X$ axis at low and high frequencies represent $R^{\dagger}$ and $R^{\text {sub }}$, respectively $\left(R^{t}-R^{\text {sub }}=R^{e}\right)$. $R^{e}$, epithelial electrical wall resistance; $R^{\text {sub }}$, subepithelial electrical wall resistance; $R^{\dagger}$, transmural wall resistance.

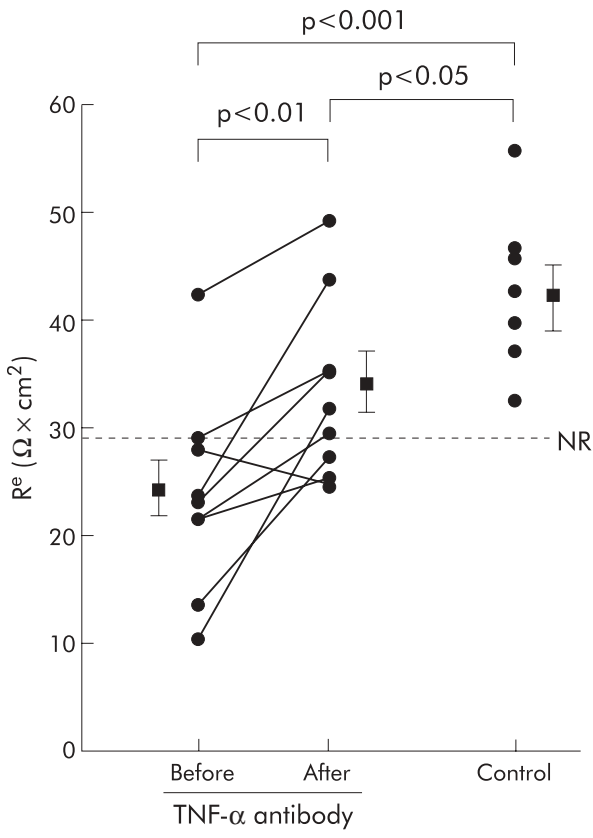

Figure 4 Epithelial electrical wall resistance $\left(R^{e}\right)$ in controls $(n=8)$ and Crohn's disease patients $(n=9)$ before and two weeks after tumour necrosis factor $\alpha$ (TNF- $\alpha$ ) antibody therapy. Data are given as means (SEM). Lower limit of the normal range (NR), as obtained from the mean of control $\pm 2 S D$, is indicated by the broken line.

patients before and after therapy, the two tailed Student's $t$ test for paired data was used. A p value of $<0.05$ was considered significant.

\section{RESULTS}

The 11 patients treated had a mean CDAI of 266 (30) before therapy which decreased after therapy to $122(21)(p<0.01)$.

\section{Epithelial apoptotic ratio}

Thin sections of sigmoid colon from eight controls and $11 \mathrm{CD}$ patients before and two weeks after infliximab were stained with TUNEL and DAPI, respectively. Enterocytes matching apoptotic criteria, as described in the methods section, were counted (fig 1). The apoptotic ratio was determined as the percentage of apoptotic enterocytes. Contribution from (apoptotic) intraepithelial lymphocytes was ruled out by analysing adjacent CD3 stained thin sections. Numerical results from TUNEL and DAPI stained sections are shown in fig 2. There was excellent correlation of results from both methods (fig 2). For this reason, apoptotic ratios obtained on TUNEL stained sections are discussed.

Table 1 Epithelial resistance $\left(R^{\mathrm{e}}\right)$, subepithelial resistance $\left(R^{\text {sub, }}\right)$ and total resistance $\left(R^{+}\right)$in controls and Crohn's disease (CD) patients

\begin{tabular}{lllll}
\hline & $\begin{array}{l}\mathbf{R}^{\mathbf{e}} \\
\left(\mathbf{\Omega} \times \mathbf{c m}^{2}\right)\end{array}$ & $\begin{array}{l}\mathbf{R}^{\text {sub }} \\
\left(\mathbf{\Omega} \times \mathbf{c m}^{2}\right)\end{array}$ & $\begin{array}{l}\mathbf{R}^{\mathbf{1}} \\
\left(\mathbf{\Omega} \times \mathbf{c m}^{2}\right)\end{array}$ & $\mathbf{n}$ \\
\hline $\begin{array}{l}\text { Control } \\
\begin{array}{l}\text { Active CD } \\
\quad \text { Before therapy }\end{array}\end{array}$ & $42(3)$ & $9(1)$ & $50(2)$ & 8 \\
$\quad$ After therapy & $24(3)^{* *}$ & $22(3)^{* *}$ & $47(3)$ & 9 \\
\hline
\end{tabular}

$R^{e}$ and $R^{\text {sub }}$ were obtained as described in the methods section ${ }^{* *} \mathrm{p}<0.01$ for $\mathrm{R}^{\mathrm{e}}$ and $\mathrm{R}^{\text {sub }}$ of pretreatment $\mathrm{CD}$ compared with controls; $t+p<0.01$ for $R^{e}$ before versus after fumour necrosis factor $\alpha$ antibody therapy. 


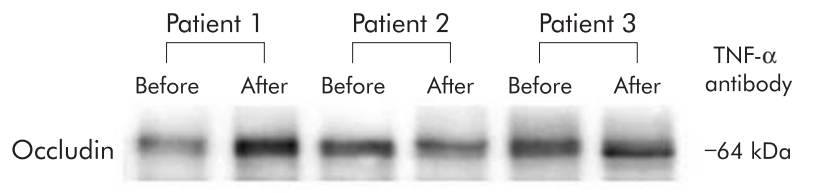

Claudin 1

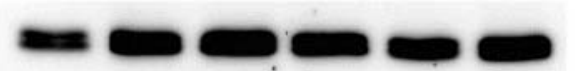

$-22 \mathrm{kDa}$

Claudin 4

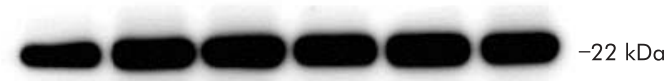

\begin{tabular}{lc} 
& After therapy \\
\hline Occludin & $109(18) \%(\mathrm{NS})$ \\
Claudin 1 & $114(25) \%(\mathrm{NS})$ \\
Claudin 4 & $108(6) \%$ (NS)
\end{tabular}

Figure 5 Expression of tight junction proteins occludin, claudin 1, and claudin 4, as obtained from crude membrane fractions in immunoblots. An original western blot is shown for three patients before and two weeks after tumour necrosis factor $\alpha$ (TNF- $\alpha$ ) antibody therapy. Statistical evaluation was performed after densitometry and is presented at the bottom. Values represent means (SEM) of protein expression analysed in comparison with pretreatment values on the same blot, which were set to $100 \%$.

There was a higher apoptotic ratio in $\mathrm{CD}$ patients $(5.3$ $(1.0) \%, \mathrm{n}=11)$ compared with controls $(2.1(0.2) \%, \mathrm{n}=8$; $\mathrm{p}<0.05)$. After therapy, the apoptotic ratio decreased to 2.9 $(1.0) \%$ in $C D(n=11 ; p<0.001)$. Thus all CD patients had an apoptotic ratio above the normal range (as defined by mean of control $( \pm 2 \mathrm{SD})$ as $1.2-3.0(\mathrm{n}=8))$ before therapy. Two weeks after TNF- $\alpha$ antibody therapy, 10 of 11 (91\%) patients reached an apoptotic ratio within the normal range.

\section{Alternating current impedance analysis}

Three original impedance plots are shown in fig 3. Epithelial resistance of two of the 11 patients could not be measured

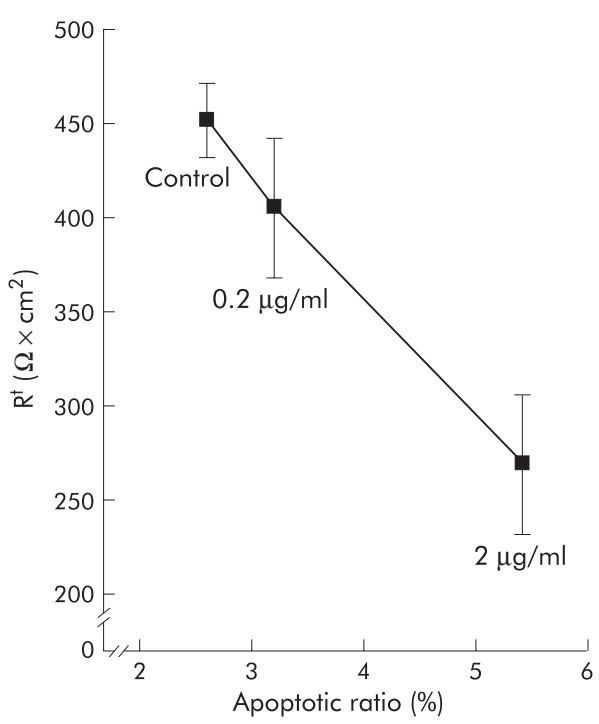

Figure 6 Correlation of apoptotic ratio and transmural resistance $\left(R^{\dagger}\right)$ in human HT-29/B6 cell lines before (Control) and after induction of apoptosis with $0.2 \mu \mathrm{g} / \mathrm{ml}$ and $2.0 \mu \mathrm{g} / \mathrm{ml}$ camptothecin for 48 hours. Late stages of apoptosis were analysed using the terminal deoxynucleotidyl transferase mediated deoxyuridine triphosphate nick end labelling (TUNEL) assay, as indicated. Data are given as means (SEM) and are representative of at least six independent experiments. due to insufficient biopsy size in one of the two biopsy procedures of the paired analysis. Data and statistical evaluation are presented in table 1 and fig 4.

In controls, epithelial resistance $\left(\mathrm{R}^{\mathrm{e}}\right)$ was 42 (3) $\Omega \times \mathrm{cm}^{2}$ $(\mathrm{n}=8)$. In $\mathrm{CD}$ before treatment, $\mathrm{R}^{\mathrm{e}}$ was lower than in controls (24 (3) $\Omega \times \mathrm{cm}^{2}, \mathrm{n}=9$; $\mathrm{p}<0.001$ compared with controls). TNF- $\alpha$ antibody therapy increased $\mathrm{R}^{\mathrm{e}}$ to 34 (3) $\Omega \times \mathrm{cm}^{2}\left(\mathrm{p}<0.01\right.$ compared with $\mathrm{R}^{\mathrm{e}}$ before therapy). Two weeks after TNF- $\alpha$ antibody therapy, six of nine patients (67\%) achieved an $\mathrm{R}^{\mathrm{e}}$ within the normal range (as defined by mean of control \pm 2 SD as $29-56 \Omega \times \mathrm{cm}^{2}(\mathrm{n}=8)$ ) (fig 4 ).

While epithelial resistance was lower in the sigmoid colon of CD patients, subepithelial resistance was increased (table 1). This may be the result of chronic inflammatory thickening of the subepithelium due to oedema and condensed collagenous fibres (fig IC). However, $\mathrm{R}^{\text {sub }}$ did not significantly change after TNF- $\alpha$ antibody therapy (table 1), even if the number of immune cells was dramatically reduced (fig 1D).

\section{Tight junction protein expression}

To investigate whether epithelial tight junction changes contribute to the increase in $\mathrm{R}^{\mathrm{e}}$ after infliximab therapy, expression of three tight junction proteins was assessed. From eight of the $11 \mathrm{CD}$ patients, sufficient protein material was obtained before as well as after therapy to perform a complete analysis of occludin, claudin 1 , and claudin 4. A representative western blot is shown in fig 5. Expression was analysed by densitometry in comparison with the pretreatment value on the same blot, which was set to $100 \%$. Compared with the pretreatment level, occludin expression was $109(18) \%(n=8)$, claudin 1 expression $114(25) \%$, and claudin 4 expression 108 (6)\% after therapy. Thus there was no significant difference between pre- and post-treatment results.

\section{Functional consequences of increased epithelial apoptoses}

To obtain more insight into the functional consequences of upregulation of epithelial apoptosis, HT-29/B6 monolayers were incubated with camptothecin. Camptothecin, at concentrations up to $10 \mu \mathrm{g} / \mathrm{ml}$, has been shown to be a selective inducer of epithelial apoptosis, leaving epithelial tight junctions unaffected. ${ }^{25}$ To obtain comparable levels of apoptosis as observed in control and CD sigmoid colon in our study, HT-29/B6 cells were studied without or with 0.2 and $2 \mu \mathrm{g} / \mathrm{ml}$ camptothecin, resulting in $2.6 \%, 3.3 \%$, and $5.4 \%$ apoptotic ratios, respectively (fig 6). As a functional consequence of this upregulation of the apoptotic rate, electrical resistance decreased from 452 (20) to 404 (37) (not significant compared with controls) and 269 (37) $\Omega \times \mathrm{cm}^{2}(\mathrm{p}<0.05$ compared with controls), respectively.

\section{DISCUSSION}

Since the 1980s several studies have shown that inflammatory changes in $\mathrm{CD}$ are paralleled by an increase in intestinal permeability. ${ }^{56} \mathrm{~A}$ recent study has indicated improvement in epithelial barrier function after TNF- $\alpha$ antibody therapy by means of in vivo permeability tests. ${ }^{11}$ In the present study, our aim was to characterise the mechanisms of the barrier effect of TNF- $\alpha$ antibody therapy in the inflamed colon of CD patients.

The re-examination interval after TNF- $\alpha$ antibody treatment was 14 days in this study, and thus analysis was not restricted to only short term effects, such as apoptosis induction. In general, intervals in studies examining immune cell apoptosis range from four hours ${ }^{16}$ to 10 weeks. ${ }^{27}$ It was shown that the proapoptotic effect of infliximab on lamina propria $\mathrm{T}$ cells extends far beyond the circulating half life of 
infliximab of 9.5 days and is still detectable 10 weeks after infliximab administration. ${ }^{27}$ Therefore, it is unlikely that an interval shorter than 14 days in our study would have led to an apoptotic ratio distinct from the present result. In contrast, it would have been impossible with a shorter interval to assess and/or exclude long term repair phenomena (for example, in respect of tight junctions).

The first important finding of our study was that epithelial apoptoses were found to be considerably upregulated compared with control sigmoid colon. An apoptotic rate of approximately $5.3 \%$ was obtained in the inflamed colon.

Thus in addition to upregulation of epithelial apoptosis in ulcerative colitis, ${ }^{28}$ this type of barrier defect is also present in CD. Comparing data reported by Strater and colleagues ${ }^{29}$ with the results of our analysis, the apoptotic rate seems to be higher in ulcerative colitis than in CD although this has to be viewed with caution as no direct comparison was performed.

The functional role of epithelial apoptosis however is still controversial. While it was originally assumed that single cell apoptosis is a regular and highly sealed process without much relevance to barrier function, our own group has obtained experimental evidence to indicate that apoptotic rosettes represent significant conductivity in epithelial HT-29/ B6 monolayers. ${ }^{7}$ Furthermore, apoptotic foci were directly shown to contribute significantly to the barrier defect in ulcerative colitis by means of a conductance scanning technique. ${ }^{10}$ However, a recent paper has questioned this by showing that addition of the caspase inhibitor ZVAD-fmk to interferon $\gamma$ plus TNF- $\alpha$ exposed T84 cells blocked apoptosis but not the decrease in resistance. ${ }^{30}$ A possible explanation for this discrepancy could be that other barrier features overruled apoptotic conductivity in T-84 cells (for example, interferon $\gamma / \mathrm{TNF}-\alpha$ induced tight junction alterations) as the reduction in resistance from 2074 to $229 \Omega / \mathrm{cm}^{2}$ indicates almost complete breakdown in barrier function in the 72 hour cytokine exposed T84 model. However, this implies that other features can influence the functional response in T84 but cannot rule out that apoptotic foci represent spots of elevated conductance. Thus taken together the contribution of epithelial apoptosis to barrier function is far from being clear and may vary from minor to predominant, depending on the particular conditions. Therefore, the functional role of epithelial apoptosis needs further investigation not only in cell models but also in the diseased mucosa, as in the present study. We believe that presenting direct experimental evidence for a parallel change in apoptotic rate and resistance without changes in tight junction proteins in membrane fractions is important experimental evidence in this respect, as a result of which the functional importance of the increase in the apoptotic rate in CD has to be estimated as rather high.

The second important finding of the present study was that TNF- $\alpha$ antibody therapy caused downregulation of the epithelial apoptotic rate in CD patients. Two weeks after treatment, 10 of 11 patients $(91 \%)$ had reached an apoptotic ratio within the normal range. This is functionally important for the colon epithelium and may represent the first step towards mucosal repair which in a subgroup of patients may even result in mucosal healing. ${ }^{31}$

The ability of TNF- $\alpha$ antibodies to downregulate epithelial apoptosis in $\mathrm{CD}$ is a priori not self evident. Taking into account the fact that immune cells in CD are eradicated via apoptotic mechanisms by TNF- $\alpha$ antibody therapy, ${ }^{14-16}$ it would have been possible that the apoptotic rate was unchanged or increased. However, in the case of epithelial cell apoptosis, neutralisation of circulating TNF- $\alpha$ together with the antibody induced reduction in the mucosal/ submucosal immune cell pool has to be assumed to play a major role in this therapy. This also means that epithelial cells in the inflamed colon of CD patients do not possess membrane bound TNF- $\alpha$ and are not targets for TNF- $\alpha$ antibodies.

That TNF- $\alpha$ antibodies can indeed normalise inflammatory increased epithelial apoptosis was recently demonstrated in SAMPI/YitFc mice, an animal model of spontaneous ileitis similar to CD in humans. ${ }^{32}$

In addition to structural barrier parameters, much effort was made in the present study to obtain functional barrier data in parallel. Measuring barrier function in tissue specimens in vitro also has the advantage of assessing function in a defined intestinal segment with a clear area reference. This enabled us to exclude influences of antibody therapy on intestinal blood circulation or motility, both of which can indirectly affect in vivo permeability tests. The result of this analysis, and our third relevant finding, was that epithelial barrier function was shown to be impaired in active CD and to be clearly improved by TNF- $\alpha$ antibody treatment.

Finally, it should be mentioned that in vitro measurements on intestinal biopsies can distinguish epithelial from subepithelial resistance by alternating current impedance analysis. This has been shown to be essential for inflamed intestine as decreased epithelial resistance is often masked by inflammatory thickening of the subepithelium as recently seen in biopsy specimens from patients with collagenous colitis for example. ${ }^{22}$ In our study, $\mathrm{R}^{\text {sub }}$ was increased in $\mathrm{CD}$ compared with controls but did not recover within the study interval of two weeks after TNF- $\alpha$ antibody treatment. The increase in $\mathrm{R}^{\mathrm{sub}}$ has to be assumed to be mainly due to altered submucosal architecture with increased thickness and composition of the subepithelial tissue layers. That such alterations do not recover within this time interval is also in accordance with another study examining histological changes four weeks after anti-TNF- $\alpha$ antibody treatment which revealed disappearance of neutrophils and a reduction in the lymphoplasmocytic infiltrate but an almost unchanged architecture. ${ }^{31}$ Furthermore, this is in line with half life values of collagens being more than 200 days, ${ }^{33}$ as a result of which much longer re-examination intervals would have been necessary to detect recovery after TNF- $\alpha$ antibody treatment, even if matrix metalloproteinase levels are elevated in CD. ${ }^{34}$ However, from a functional point of view, altered subepithelial tissue in $C D$ is less important as in contrast with the collagenous band in collagenous colitis, capillaries penetrate most of these subepithelial tissue layers in CD mucosa and are located in close proximity to the epithelium. Thus these morphological changes are of minor importance for solute reabsorption and the pathogenesis of diarrhoea in $\mathrm{CD}$.

In $C D$, changes in tight junction structure have been detected by morphological studies using freeze fracture electron microscopy ${ }^{35} 36$ as well as by protein biochemical studies on tight junction molecule expression..$^{37}$ In the latter study, occludin expression was found to be reduced compared with controls whereas claudin 1 was not significantly affected. The fourth important result of our study was that a direct comparison between pretreatment protein expression levels and expression levels two weeks after TNF- $\alpha$ antibody treatment revealed no significant change in tight junction protein expression for occludin, claudin 1 , or claudin 4.

As epithelial apoptosis and tight junction protein expression are not the only barrier determining structures, the question arose of whether or not changes in epithelial resistance in $\mathrm{CD}$ before and after therapy can be (fully) explained by altered apoptotic ratios. Thus we induced apoptoses in HT-29/B6 cell monolayers with the topoisomerase inhibitor camptothecin. ${ }^{25}$ Although this cell model represents only an approximation of the complex mucosal architecture of the native colon, it allows adjustment of apoptotic rates to levels detected in the human colon of 
controls or CD patients. Furthermore, HT-29/B6 cells obtained from a colonic cancer are also an intermediate tight epithelium and possess all signal transduction pathways relevant for intestinal barrier regulation during inflammation, including cytokine dependent modulation of tight junctions and apoptoses. In addition, the same staining and counting procedures were applied in a parallel design. An increasing apoptotic ratio from $2.6 \%$ to $5.4 \%$, which reflects the colonic epithelium of controls and CD patients before therapy, caused a decrease in epithelial resistance of $-40 \%$. This is almost exactly the same as that observed for the decrease in epithelial resistance in untreated CD patients compared with controls, which was $-43 \%$. Therefore, it seems reasonable to conclude that upregulation of epithelial apoptosis was the predominant factor in barrier dysfunction in Crohn's colitis in our study.

Disturbance in epithelial barrier function in intestinal inflammation has two important consequences. On the one hand, it contributes to diarrhoea by a leak flux mechanism. ${ }^{822}$ On the other hand, it enables antigens to be taken up and contributes to perpetuation of the inflammatory process. The former functional consequence could be relevant for the observation that diarrhoea in some CD patients stops 24-48 hours after TNF- $\alpha$ antibody treatment, a time period which could allow barrier improvement as a result of downregulation of epithelial apoptosis. That apoptotic events in the epithelium are indeed functionally important is supported by direct experimental evidence. An increase in the apoptotic ratio to $12 \%$ in HT-29/B6 colonic model epithelium switched the intermediate tight into a leaky type epithelium. ${ }^{25}$ Therefore, one has to assume that the increase in apoptotic ratio in $\mathrm{CD}$ patients of up to $15 \%$ has severe functional implications.

Epithelial barrier repair in CD due to TNF- $\alpha$ antibody treatment, possibly resulting in a decrease in antigen uptake, has to be assumed to be more important for the long term anti-inflammatory therapy effect, and indeed "barrier healing" could represent the first step towards "mucosal healing" observed in some of patients under continuous TNF- $\alpha$ antibody therapy.

In conclusion, we have shown a significant decrease in epithelial cell apoptosis in CD patients after TNF- $\alpha$ antibody therapy which was accompanied by an increase in epithelial resistance. Tight junction protein expression did not contribute to this barrier repair.

\section{ACKNOWLEDGEMENTS}

This study was supported by grants from Deutsche Forschungsgemeinschaft (DFG Schu 559/6-3 and Schu 559/7-1), the German $\mathrm{BMBF} / \mathrm{DLR}$ in the medical competence network inflammatory bowel disease, and from the Else Kroener-Fresenius-Stiftung. The excellent assistance of Anja Fromm and Susanna Schon and the great support of the electronic engineer Detlef Sorgenfrei is gratefully acknowledged.

\section{Authors' affiliations}

S Zeissig*, C Bojarski", N Buergel, J Mankertz, M Zeitz, J D Schulzke, Departments of Gastroenterology, Infectious Diseases, and

Rheumatology, Charité Campus Benjamin Franklin, Berlin, Germany

M Fromm, Department of Clinical Physiology, Charité Campus Benjamin Franklin, Berlin, Germany

*S Zeissig and C Bojarski contributed equally to this work.

\section{REFERENCES}

1 Prantera C, Cottone M, Pallone F, et al. Mesalamine in the treatment of mild to moderate active Crohn's ileitis: results of a randomized, multicenter trial. Gastroenterology 1999;116:521-6.
2 van Dullemen HM, van Deventer SJ, Hommes DW, et al. Treatment of Crohn's disease with anti-tumor necrosis factor chimeric monoclonal antibody (cA2). Gastroenterology 1995; 109:129-35.

3 Hanaver SB, Feagan BG, Lichtenstein GR, et al. Maintenance infliximab for Crohn's disease: the ACCENT I randomised trial. Lancet 2002;359:1541-9.

4 Sanderson IR, Boulton P, Menzies I, et al. Improvement of abnormal lactulose/ rhamnose permeability in active Crohn's disease of the small bowel by an elemental diet. Gut 1987;28:1073-6.

5 Bjarnason I, MacPherson A, Hollander D. Intestinal permeability: an overview. Gastroenterology 1995;108:1566-81.

6 MacDonald TT, Hutchings $\mathrm{P}$, Choy MY, et al. Tumour necrosis factor-alpha and interferon-gamma production measured at the single cell level in normal and inflamed human intestine. Clin Exp Immunol 1990;81:301-5.

7 Gitter AH, Bendfeldt K, Schulzke JD, et al. Leaks in the epithelial barrier caused by spontaneous and TNF-alpha-induced single-cell apoptosis. FASEB J 2000; 14:1749-53.

8 Schmitz H, Fromm M, Bentzel CJ, et al. Tumor necrosis factor-alpha (TNFalpha) regulates the epithelial barrier in the human intestinal cell line HT29/B6. J Cell Sci 1999; 1 12(Pt 1):137-46.

9 Mankertz J, Tavalali S, Schmitz H, et al. Expression from the human occludin promoter is affected by tumor necrosis factor alpha and interferon gamma. J Cell Sci 2000;113(Pt 11):2085-90.

10 Gitter AH, Wullstein F, Fromm M, et al. Epithelial barrier defects in ulcerative colitis: characterization and quantification by electrophysiological imaging. Gastroenterology 2001;121:1320-8.

11 Suenaert P, Bulteel V, Lemmens L, et al. Anti-tumor necrosis factor treatment restores the gut barrier in Crohn's disease. Am J Gastroenterol 2002;97:2000-4

12 Bu P, Keshavarzian A, Stone DD, et al. Apoptosis: one of the mechanisms that maintains unresponsiveness of the intestinal mucosal immune system. $\mathrm{J}$ Immunol 2001; 166:6399-403.

13 Boirivant M, Marini M, Di Felice G, et al. Lamina propria T cells in Crohn's disease and other gastrointestinal inflammation show defective CD2 pathwayinduced apoptosis. Gastroenterology 1999;1 16:557-65.

14 Ina K, Itoh J, Fukushima K, et al. Resistance of Crohn's disease T cells to multiple apoptotic signals is associated with a $\mathrm{Bcl}-2 /$ Bax mucosal imbalance. $\mathrm{J}$ Immunol 1999;163:1081-90.

15 ten Hove T, van Montfrans C, Peppelenbosch MP, et al. Infliximab treatment induces apoptosis of lamina propria T lymphocytes in Crohn's disease. Gut 2002; 50:206-11.

16 Lugering A, Schmidt M, Lugering $N$, et al. Infliximab induces apoptosis in monocytes from patients with chronic active Crohn's disease by using a caspase-dependent pathway. Gastroenterology 2001;121:1145-57.

17 Best WR, Becktel JM, Singleton JW. Rederived values of the eight coefficients of the Crohn's disease activity index (CDAl). Gastroenterology 1979:77(Pt 2):843-6.

18 Kapuscinski J. DAPI: a DNA-specific fluorescent probe. Biotech Histochem 1995;70:220-33

19 Stockmann M, Fromm M, Schmitz H, et al. Duodenal biopsies of HIV-infected patients with diarrhoea exhibit epithelial barrier defects but no active secretion. AIDS 1998;12:43-51.

20 Fromm M, Schulzke JD, Hegel U. Control of electrogenic Na+ absorption in rat late distal colon by nanomolar aldosterone added in vitro. Am J Physiol 1993;264(Pt 1):E68-73.

21 Schmitz $\mathrm{H}$, Fromm $\mathrm{M}$, Bode $\mathrm{H}$, et al. Tumor necrosis factor-alpha induces $\mathrm{Cl}$ and $\mathrm{K}+$ secretion in human distal colon driven by prostaglandin E2. Am J Physiol 1996;271(Pt 1):G669-74.

22 Burgel N, Bojarski C, Mankertz J, et al. Mechanisms of diarrhea in collagenous colitis. Gastroenterology 2002;123:433-43.

23 Gitter AH, Schulzke JD, Sorgenfrei D, et al. Ussing chamber for highfrequency transmural impedance analysis of epithelial tissues. J Biochem Biophys Methods 1997;35:81-8.

24 Kreusel KM, Fromm M, Schulzke JD, et al. Cl- secretion in epithelial monolayers of mucus-forming human colon cells (HT-29/B6). Am J Physiol 1991;261(Pt 1):C574-82.

25 Bojarski C, Gitter AH, Bendfeldt K, et al. Permeability of human HT-29/B6 colonic epithelium as a function of apoptosis. J Physiol 2001;535(Pt 2): 541-52.

26 Bjarnason I, O'Morain C, Levi AJ, et al. Absorption of 51 chromium-labeled ethylenediaminetetraacetate in inflammatory bowel disease. Gastroenterology 1983;85:318-22.

27 Di Sabatino A, Ciccocioppo R, Cinque B, et al. Defective mucosal T cell death is sustainably reverted by infliximab in a caspase dependent pathway in Crohn's disease. Gut 2004;53:70-7.

28 Hagiwara C, Tanaka M, Kudo H. Increase in colorectal epithelial apoptotic cells in patients with ulcerative colitis ultimately requiring surgery. J Gastroenterol Hepatol 2002; 17:758-64.

29 Strater J, Wellisch I, Riedl S, et al. CD95 (APO-1/Fas)-mediated apoptosis in colon epithelial cells: a possible role in ulcerative colitis. Gastroenterology 1997;113:160-7.

30 Bruewer $M$, Luegering $A$, Kucharzik T, et al. Proinflammatory cytokines disrupt epithelial barrier function by apoptosis-independent mechanisms. $\mathrm{J}$ Immunol 2003;171:6164-72.

31 D'Haens G, Van Deventer S, Van Hogezand R, et al. Endoscopic and histological healing with infliximab anti-tumor necrosis factor antibodies in Crohn's disease: A European multicenter trial. Gastroenterology 1999;116:1029-34.

32 Marini M, Bamias G, Rivera-Nieves J, et al. TNF-alpha neutralization ameliorates the severity of murine Crohn's-like ileitis by abrogation of intestinal epithelial cell apoptosis. Proc Natl Acad Sci U S A 2003;100:8366-71. 
33 Rucklidge GJ, Milne G, McGaw BA, et al. Turnover rates of different collagen types measured by isotope ratio mass spectrometry. Biochim Biophys Acta 1992; 1156:57-61.

34 von Lampe B, Barthel B, Coupland SE, et al. Differential expression of matrix metalloproteinases and their tissue inhibitors in colon mucosa of patients with inflammatory bowel disease. Gut 2000:47:63-73.

35 Marin ML, Geller SA, Greenstein AJ, et al. Ultrastructural pathology of Crohn's disease: correlated transmission electron microscopy, scanning electron microscopy, and freeze fracture studies. Am J Gastroenterol 1983;78:355-64

36 Marin ML, Greenstein AJ, Geller SA, et al. A freeze fracture study of Crohn's disease of the terminal ileum: changes in epithelial tight junction organization. Am J Gastroenterol 1983:78:537-47.

37 Kucharzik T, Walsh SV, Chen J, et al. Neutrophil transmigration in inflammatory bowel disease is associated with differential expression of epithelial intercellular junction proteins. Am J Pathol 2001;159:2001-9

\title{
EDITOR'S QUIZ: GI SNAPSHOT
}

\author{
Jaundice and anaemia
}

Robin Spiller, Editor

\section{Clinical presentation}

A 50 year old woman presented with progressive jaundice and shortness of breath. She had no history of blood transfusions or recent travel. She had been taking more than $120 \mathrm{~g}$ of alcohol per day for 20 years. Her family history was unremarkable. On examination, jaundice, oedema, splenomegaly, and flapping tremor were notable. Laboratory values were as follows: leucocytes $3.8 \times 10^{9} /$; haemoglobin $5.6 \mathrm{~g} / \mathrm{dl}$; platelet count $33 \times 10^{9} / \mathrm{l}$; alanine aminotransferase $49 \mathrm{IU} / \mathrm{l}$; aspartate aminotransferase $16 \mathrm{IU} / \mathrm{l}$; lactate dehydrogenase $439 \mathrm{IU} / \mathrm{l}$; total bilirubin $15.7 \mathrm{mg} / \mathrm{dl}$; indirect bilirubin $11.4 \mathrm{mg} / \mathrm{dl}$; albumin $2.9 \mathrm{~g} / \mathrm{dl}$; cholesterol $205 \mathrm{mg} / \mathrm{dl}$; ferritin $968 \mu \mathrm{g} / \mathrm{l}$; and prothrombin activity $28 \%$. Tests for hepatitis B surface antigen, anti-hepatitis $\mathrm{C}$ virus antibody, antinuclear antibody, and antimitochondrial antibody were negative.

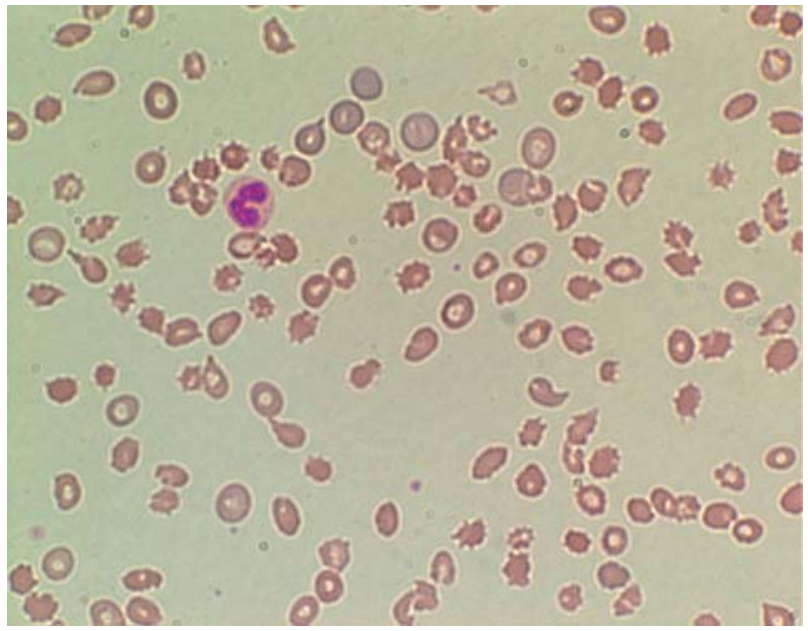

Figure 1 Peripheral blood smear.

\section{Question}

A peripheral blood smear (fig 1) and an abdominal T2 weighed magnetic resonance image (fig 2) are depicted. What is the diagnosis?

See page 1308 for answer

This case is submitted by:

T Makishi, A Hokama, H Sakugawa, T Nakayoshi, T Maeshiro, K Maeda, J Shiroma, T Oshiro, F Kinjo, A Saito First Department of Internal Medicine, University of the Ryukyus, Okinawa, Japan

Correspondence to: $\operatorname{Dr}$ A Hokama, First Department of Internal Medicine, University of the Ryukyus, 207 Uehara, Nishihara, Okinawa 903-0215, Japan; hokama-a@med.u-ryukyu.ac.jp doi: $10.1136 /$ gut.2003.034785

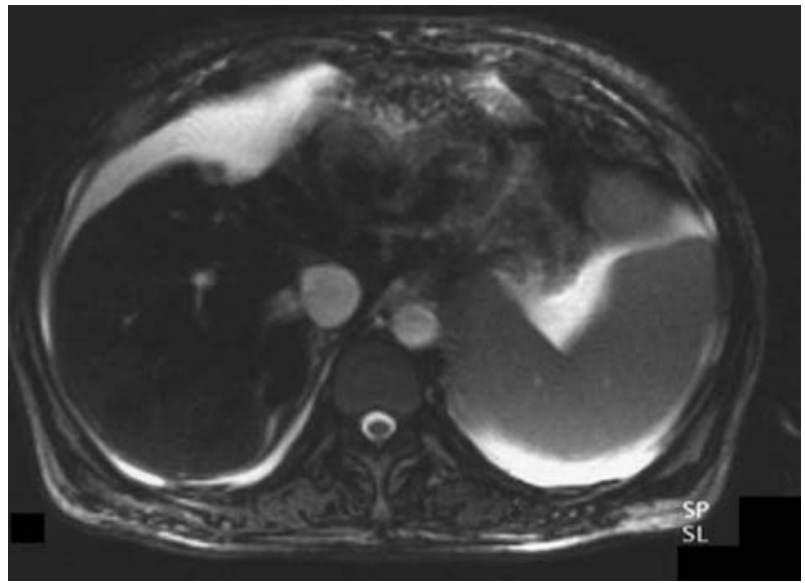

Figure 2 Abdominal T2 weighed magnetic resonance image. 